\title{
Dr. Beat Richners Einsatz für Frieden und Wiedergutmachung
}

\section{David Holzmann}

Prof. Dr. med

\author{
Wer in der Schweiz kennt nicht Dr. Beat Richner, Ehrendoktor der Universität Lau- \\ sanne und Zürich? Seine Projekte wurden von der School of Hygiene \& Tropical \\ Medicine in London als «best project ever seen» beurteilt. Die Meriten können und \\ sollen hier nicht aufgezählt werden, sie sind im Buch seines Freundes Peter Rothen- \\ bühler sorgfältig dokumentiert. Es stellt sich die Frage, warum Richners Leistung \\ nicht einen Nobelpreis wert war?
}

Die Antwort wird verständlich, wenn man sich Richners Grundintention vor Augen führt. "Was wir hier tun, ist weder eine Hilfe noch eine Unterstützung, es ist nur Wiedergutmachung. Die Probleme der 70er Jahre wurden durch schreckliche Fehlentscheide des Westens erzeugt, die man nie mehr auslöschen kann.» Er verstand sein Werk als Wiederherstellung; dabei strafte er seine Widersacher mit Verachtung. «Der menschliche Körper ist überall der gleiche, die Medizin muss auch überall gleich sein, wir machen nicht arme Medizin für arme Menschen.» Wenn Richner die Protagonisten wie die WHO, die für eine «arme Medizin für arme Menschen» oder eben "Basismedizin» eintraten, als «letzte Bastionen des MarxismusLeninismus» titulierte, scheint das harter Tobak zu sein. Doch er begründete diesen Vorwurf damit, dass unter Mao, Pol Pot und anderen Regimes hohe Funktionäre sich in amerikanischen Top-Kliniken behandeln liessen, während für das arme Volk «Basismedizin» bzw. «arme Medizin» genügen musste.

Die WHO kritisierte er wegen deren Essential Drug List, auf welcher immer noch das Antibiotikum Chloramphenicol figuriert, das wegen seiner hämatopoetischen Nebenwirkungen alljährlich zu 80 Todesfällen in seinen Spitälern führte. Wenn die gleiche WHO behauptete, «Tuberkulose sei bei Kindern kein Problem, und ausserdem sei die Krankheit bei Kindern nicht ansteckend", dann gehöre das an den Internationalen Strafgerichtshof, so Richner. Den Vorwurf, Richner betreibe Luxusmedizin, bezeichnete Bundesrat Delamuraz im Jahre 1996 als «Diskriminierung und Neokolonialismus».

Ein bis heute aktueller Kritikpunkt richtete Richner an die Adresse der Pharmaindustrie. Trotz Kauf von Antibiotika und computerisierten Laborautomaten kamen die Firmen ihm im Preis nicht entgegen. Richner scheute sich nicht, den ersten Vizeminister im kambodschanischen Gesundheitsministerium zu denunzieren, indem er dessen Arbeit für die Firma Roussel offenlegte, den Hauptproduzenten und Hauptlieferanten des in den Industrieländern für Kinder verbotenen Antibiotikums Chloramphenicol.

Richners Kritik an der DEZA (Direktion für Entwicklung und Zusammenarbeit), obschon diese den grössten Betrag ihres Budgets für seine Projekte ausgab, begründet er mit der oben erwähnten Idee der Wiedergutmachung und der «Force humanitaire», die er schon

Medizin muss überall gleich sein, wir machen nicht arme Medizin für arme Menschen.

als Medizinstudent verfolgte. Deswegen muss die offizielle Schweiz sich voll und ganz hinter seine Projekte stellen und letztlich finanziell tragen. Damit steht Richner in der humanitären Tradition unseres Landes, als aktive Hilfe vor Ort und Prävention gegen Flüchtlingsströme. Sein nachhaltiges Konzept ist ein Leuchtturm der wahren Wiedergutmachung, eine Aufarbeitung von kriegerischen Vernichtungsfeldzügen und der modernen Form des Kolonialismus.

Richner war kein Diplomat. Seine grundlegende Kritik lieferte Stoff für viele Gegner, die ihn in Leserbriefen und Artikeln von Tages-Anzeiger und NZZ zurechtwiesen und potentielle Spender abhalten wollten. Der Erfolg war aber derart eindrücklich, dass allein die jährlich veröffentlichten Zahlen alle Kritiker Lügen straften: In den fünf Spitälern und einer Maternité, mit einem Jahresbudget von 42 Millionen, wurden 16,3 Millionen Kinder seit 1992 unentgeltlich behandelt. Richners Ma- 
nagement war so klein, dass es unter seinem Arm Platz hatte, wogegen andere Spitäler ganze Stockwerke dafür beanspruchen. Der Betrieb seiner Spitäler hat Vorbildcharakter für uns. Richner hat vorgemacht, was es braucht, um sinnvolle Medizin, patientenorientierte Behandlung und echte Prävention zu betreiben. Mehr noch, er hat gezeigt, wie wir Ärzte einen Beitrag zum Weltfrieden leisten können.

\section{Warum ist Beat Richner ein Vorbild, was zeigt uns sein Leben?}

1. Es ist ein Menschenrecht, dass jeder Erdenbürger Zugang zur modernen Medizin haben muss.

2. Die Behandlung einer Krankheit richtet sich nach medizinisch-wissenschaftlichen Kriterien und nicht nach der politischen Ausrichtung, Religion oder Herkunft des Patienten. Als Arzt muss man sich als Anwalt und Hilfeleister seiner Patienten verstehen.

3. Der finanzielle Aufwand in der medizinischen Versorgung könnte drastisch gesenkt werden, wenn die ganze Administration auf das medizinisch Notwendige reduziert würde, Pharmaunternehmen sowie Medtech-Firmen ihre Preise stark reduzieren würden und bei den Geldflüssen keine Gelder in Taschen von Funktionären abgezweigt würden.

4. Um der Korruption in der medizinischen Versorgung zuvorzukommen, sind verschiedene Massnahmen notwendig. In den Gesundheitsberufen braucht es einen angemessenen Lohn für das Personal. Spenden und Zuwendungen dürfen nicht zweckentfremdet werden. Es sollte unmöglich sein, exorbitante Zusatzverdienste auf Basis ökonomiegesteuerter «Anreize» zu erwerben, die ethisch ohnehin zu hinterfragen sind. Sofern vorhanden, müssen Interessenkonflikte offengelegt werden. Den grössten Gewinn und vollendete berufliche Genugtuung kann jeder Arzt durch seinen Einsatz für das Wohl seiner Patienten ernten; es gilt, dieser ethischen Verpflichtung in Erziehung, Aus- und Weiterbildung höchste Bedeutung beizumessen.

5. Die Verpflichtung, für die Gesundheit der Menschen zu sorgen, ist in jedem Fall höher zu gewichten als politische und wirtschaftliche Interessen. Um dieser Verpflichtung nachkommen zu können, braucht der Arzt Charakterstärke und Unabhängigkeit, aber auch Bescheidenheit und Demut. Sich für die Gesundung von kranken Menschen einzusetzen muss ein grösserer Ansporn sein als der eigene Ruf, das Ansehen und letztlich auch die Angst vor ungerechtfertigten Anfeindungen.
6. Der Arztberuf erfordert die Bereitschaft und Fähigkeit, sich mit Kollegen und anderen Berufsgruppen frei von Überheblichkeit und Missgunst zu vernetzen. Gefordert ist ein würdiger und wertschätzender Umgang mit allen Mitarbeitern und Berufsgruppen, die sich als Team verstehen und für die gleichen Ziele eintreten.

7. Arzt sein bedeutet, sich in das politische Geschehen einmischen zu dürfen, heisst auch, sich gegen den Krieg zu stellen und sich für den Frieden als oberstes Ziel der Menschheit einzusetzen. Wo Menschen durch Kriege zu Schaden kommen, muss der behandelnde Arzt von den politisch Verantwortlichen Unterstützung in Form von Wiedergutmachungen einfordern. Spätestens ab diesem Punkt kann der Arzt nicht mehr diplomatisch sein, er ist vielmehr Akteur mit konkreten Zielen (Frieden) und Visionen (Gerechtigkeit).

8. Der dem humanitären Prinzip verpflichtete Arzt hilft auch seinem Widersacher, wenn dieser krank oder verletzt ist, streng nach den Prinzipien Henri Dunants. Die konsequente und bedingungslose Hilfe kann unter Umständen den Kontrahenten zum Einlenken, zur Einsicht bewegen. Spätes Einlenken von früheren Widersachern soll nicht nachtragend immer wieder thematisiert werden. Es gilt, die Gemeinsamkeiten im Hier und Jetzt zu pflegen und weiterzuschreiten.

9. Es ist nicht ungefährlich, sich konsequent für Wiedergutmachung, Versöhnung und Frieden einzusetzen. Die heftigen Anfeindungen muss man aushalten können.

Beat Richners Leistung und Werk ist für jeden Mediziner, ob hier oder in Kambodscha, ein Lehrstück. Sein Lebenswerk ist seine Anstrengung für den Frieden, die Versöhnung und die Wiedergutmachung, was offenbar verschiedenen Mächtigen dieser Welt missfiel.

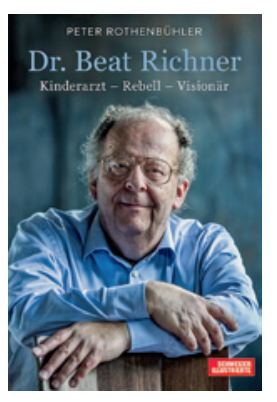

Peter Rothenbühler:

Dr. Beat Richner.

Kinderarzt - Rebell - Visionär,

Zürich: Beobachter; 2019.

Siehe auch die Buchbesprechung von Gabriella Hunziker: Schweiz Ärzteztg. 2020;101(6):189. 